\title{
On implicit Lagrangian differential systems
}

\author{
by S. JANECZKo (Warszawa)
}

Bogdan Ziemian in memoriam

\begin{abstract}
Let $(P, \omega)$ be a symplectic manifold. We find an integrability condition for an implicit differential system $D^{\prime}$ which is formed by a Lagrangian submanifold in the canonical symplectic tangent bundle $(T P, \dot{\omega})$.
\end{abstract}

1. Introduction. Let $P$ be a smooth manifold. A submanifold $D^{\prime}$ of the tangent bundle $T P$ is considered as a first order differential equation. If $D^{\prime}$ is not transversal to the tangent bundle fibration then $D^{\prime}$ is called an implicit differential equation. This type of equation may be written locally in the form

$$
\dot{x}_{i}=g_{i}(x, \lambda), \quad 0=f_{j}(x, \lambda),
$$

where $g_{i}, f_{j}: \mathbb{R}^{n} \times \mathbb{R}^{k} \rightarrow \mathbb{R}(1 \leq i \leq n, 1 \leq j \leq k)$ are smooth functions and the parameter $\lambda$ cannot be "eliminated" from the second equation (cf. [12]). There is an interesting question concerning the local integrability of such equations (cf. $[2,12,13,7]$ ).

As an example we can take the equation

$$
\dot{x}=\lambda, \quad 0=x+(\lambda-a)^{2},
$$

where $(\dot{x}, x) \in T \mathbb{R}$. We see that $D^{\prime}$ is a parabola tangent to the fiber of $T \mathbb{R}$ at $(a, 0)$ and no differentiable solution of the above equation can pass through $(a, 0)$ unless $a=0$. This observation suggests that there are some local properties of the singularity set of $D^{\prime}$, i.e. the set of singular points of the projection $\left.\tau_{P}\right|_{D^{\prime}}: D^{\prime} \rightarrow P(\mathrm{cf}$. $[1,8])$, where $\tau_{P}: T P \rightarrow P$ is the tangent bundle projection, that assure integrability.

2000 Mathematics Subject Classification: Primary 58C27; Secondary 57R45, 70H15.

Key words and phrases: symplectic manifold, Lagrangian manifold, integrability, implicit differential equation.

Partially supported by KBN grant Nr 2 P03A 02017. 
In this note we formulate the integrability problem for generalized Hamiltonian systems (cf. [3]), i.e. for $D^{\prime}$ being Lagrangian in the canonical symplectic structure of $T P$ if $P$ is symplectic. We prove a necessary condition and a sufficient condition for complete integrability and show their connection to abnormal curves in the geometry of distributions.

2. Implicit differential systems. Let $(P, \omega)$ be a symplectic manifold. The tangent bundle $T P$ is isomorphic to the cotangent bundle $T^{*} P$ by the vector bundle morphism $\beta: T P \ni u \rightarrow u\rfloor \omega \in T^{*} P$. Let $\pi_{P}: T^{*} P \rightarrow P$ be the cotangent bundle projection and $\theta_{P}$ be the Liouville one-form on $T^{*} P$. The corresponding canonical symplectic structure on $T P$ is defined by

$$
\tau_{P}=\pi_{P} \circ \beta, \quad \kappa=\beta^{*} \theta_{P},
$$

and we define

$$
\dot{\omega}=d \kappa=\beta^{*} d \theta_{P}
$$

Let $\left(x_{i}, y_{j}\right), 1 \leq i, j \leq n, \operatorname{dim} P=2 n$, be local coordinates on $P$ such that

$$
\omega=\sum_{i=1}^{n} d y_{i} \wedge d x_{i} .
$$

We use the canonical coordinates $\left(x_{i}, y_{j}, \dot{x}_{k}, \dot{y}_{l}\right), 1 \leq i, j, k, l \leq n$, on $(T P, \dot{\omega})$ and write $\kappa$ in these coordinates:

$$
\kappa=\sum_{i=1}^{n}\left(\dot{y}_{i} d x_{i}-\dot{x}_{i} d y_{i}\right) .
$$

Definition 2.1. A submanifold $D^{\prime}$ of $T P$ is called a first order differential system. A differentiable mapping $\gamma: I \rightarrow P$ is called an integral curve of $D^{\prime}$ if the vector $\dot{\gamma}(t)$ tangent to $\gamma$ at $\gamma(t)$ belongs to $D^{\prime}$ for each $t \in I=[\alpha, \beta]$. A differential system $D^{\prime} \subset T P$ is said to be integrable (completely integrable) if for each element $u \in D^{\prime}$ there is an integral curve $\gamma$ of $D^{\prime}$ such that $\dot{\gamma}(0)=u$.

Definition 2.2. A first order differential system $D^{\prime}$ is called an infinitesimal symplectic relation or integrable Lagrangian differential system if it is integrable and is a Lagrangian submanifold of the symplectic space $(T P, \dot{\omega})$. If, at some point $u \in D^{\prime}$, the Lagrangian differential system (i.e. Lagrangian submanifold of $(T P, \dot{\omega})) D^{\prime}$ is not transversal to the fibre $T_{\tau_{P}(u)} P$, then $D^{\prime}$ is also called an implicit Lagrangian differential system.

Any Lagrangian submanifold of $(T P, \dot{\omega})$ is locally generated by a generating family (Morse family [14]) in the cotangent bundle structure $\left(T P, \tau_{P}, \kappa\right)$ on $T P$. In local terms this means that there is an open neighbourhood of every point of $D^{\prime}$ such that in the above introduced Darboux coordinates 
around the chosen point, the differential system $D^{\prime}$ is described by the following system of equations, where $\left.\kappa\right|_{D^{\prime}}=-d F$ :

$$
\begin{aligned}
\dot{x}_{i} & =\frac{\partial F}{\partial y_{i}}(x, y, \lambda), & 1 & \leq i \leq n, \\
\dot{y}_{j} & =-\frac{\partial F}{\partial x_{j}}(x, y, \lambda), & 1 & \leq j \leq n, \\
0 & =\frac{\partial F}{\partial \lambda_{k}}(x, y, \lambda), & 1 & \leq k \leq m,
\end{aligned}
$$

where $F: \mathbb{R}^{2 n} \times \mathbb{R}^{m} \rightarrow \mathbb{R}$ is a smooth function in a neighbourhood $\mathcal{O}$ of zero in $\mathbb{R}^{2 n} \times \mathbb{R}^{m}$.

EXAMPLE 2.1. Let $X$ be a connected smooth manifold, $\operatorname{dim} X=n$, and let $V$ be a smooth $k$-dimensional distribution on $X$. Let $\left\{X_{1}, \ldots, X_{k}\right\}$ be a local basis of vector fields generating $V$. We say that $V$ satisfies the Hörmander condition at $x \in X$ if these vector fields together with all their commutators span $T_{x} X$. If this condition is satisfied at every point of $X$ then $V$ is called a nonholonomic distribution (cf. [10, 4, 5, 11]). We denote by $\Omega_{V}$ the space of horizontal (absolutely continuous) curves $\gamma:[0,1] \rightarrow X$ $(\dot{\gamma} \in V)$. The subset of all paths starting at $x_{0} \in X$ is denoted by $\Omega_{V}\left(x_{0}\right)$. The endpoint map

$$
\operatorname{end}_{x_{0}}: \Omega_{V}\left(x_{0}\right) \rightarrow X
$$

assigns to each curve its endpoint $\operatorname{end}_{x_{0}}(\gamma)=\gamma(1)$. The horizontal curve $\gamma \in \Omega_{V}$ is called singular if it is a singular point of the map end ${ }_{\gamma(0)}$. The singular curves are integral curves of the implicit Lagrangian differential system $D^{\prime}$ generated by

$$
\begin{aligned}
F: T^{*} X \times \mathbb{R}^{k} & \rightarrow \mathbb{R}, \quad F(x, y, \lambda)=\sum_{i=1}^{k} \lambda_{i}\left\langle y, X_{i}(x)\right\rangle, \\
\dot{x}_{j} & =\sum_{i=1}^{k} \lambda_{i} X_{i j}(x), \\
\dot{y}_{j} & =-\sum_{i=1}^{k} \lambda_{i}\left\langle y, \frac{\partial X_{i}}{\partial x_{j}}(x)\right\rangle, \\
0 & =\left\langle y, X_{i}(x)\right\rangle,
\end{aligned}
$$

where $X_{i}=\sum_{j=1}^{n} X_{i j} \partial / \partial x_{j}, 1 \leq j \leq n, 1 \leq i \leq k$.

The integrability of this system will be considered in the next section. A sufficient condition for integrability will be a specialized version of the corresponding integrability theorem 3.1.

ExAMPLE 2.2. Let $X$ be the 4-dimensional space-time manifold of general relativity (cf. [11]). The cotangent bundle $T^{*} X$ represents the phase space of 
a relativistic particle of mass $m$. Its dynamics is represented by the implicit Lagrangian differential system $D^{\prime} \subset T\left(T^{*} X\right)$ generated by the generating family

$$
F(x, y, \lambda)=\lambda\left(\left(\sum_{i, j=1}^{4} g^{i j} y_{i} y_{j}\right)^{1 / 2}-m\right),
$$

where $g^{i j}$ denote the components of the contravariant metric tensor. We easily see, by Proposition 3.2, that this system is integrable.

3. Integrability condition. Let $D^{\prime}$ be a first order differential system, $\operatorname{dim} D^{\prime}=\operatorname{dim} P$. If $D^{\prime}$ is Lagrangian then we denote by $C_{D^{\prime}}$ the set of critical values of the projection $\tau_{P}: D^{\prime} \rightarrow P$. We call this set the differential (or Hamiltonian) caustic of $D^{\prime}$. By a straightforward check we obtain the following necessary condition for integrability of $D^{\prime}$ :

Proposition 3.1. Assume $D^{\prime}$ is an integrable Lagrangian differential system and $C_{D^{\prime}}$ is a stratified set. Then

$$
T C_{D^{\prime}}^{k} \subset D^{\prime}, \quad k \in J
$$

where $T C_{D^{\prime}}^{k}$ is the tangent bundle to the stratum $C_{D^{\prime}}^{k}$ and $C_{D^{\prime}}=\bigcup_{k \in J} C_{D^{\prime}}^{k}$.

Now we formulate a sufficient condition for integrability. Let $M_{\mathrm{s}}$ denote the space of all $m \times m$ symmetric matrices over $\mathbb{R}$. For each $r \in \mathbb{N} \cup\{0\}$, let $\Sigma_{r}$ denote the subset of $M_{\mathrm{S}}$ consisting of all matrices of rank $r$. Then $\Sigma_{r}$ is a submanifold of $M_{\mathrm{s}}$ of codimension

$$
\frac{(m-r)(m-r+1)}{2} .
$$

We view $H(x, y, \lambda)=\left(\frac{\partial^{2} F}{\partial \lambda^{k} \partial \lambda^{l}}\right)(x, y, \lambda)$ as a smooth mapping of $\mathcal{O}$ into $M_{\mathrm{s}}$. Also we denote by $E_{s}^{\mathcal{O}}$ the space of all smooth mappings of $\mathcal{O}$ into $M_{\mathrm{S}}$ endowed with the $C^{\infty}$-Whitney topology.

Let $D^{\prime} \subset(T P, \dot{\omega})$ be a Lagrangian submanifold.

THEOREM 3.1. Assume that for every point of $D^{\prime}$ there exists a generating Morse family $F: \mathbb{R}^{2 n} \times \mathbb{R}^{m} \supset \mathcal{O} \ni(x, y, \lambda) \mapsto F(x, y, \lambda) \in \mathbb{R}$ such that

(i) $H: \mathcal{O} \rightarrow M_{\mathrm{s}}$ is transverse to all $\Sigma_{r}, r=1, \ldots, m$ (i.e. $H$ is a generic mapping in the space $E_{\mathrm{s}}^{\mathcal{O}}$ ).

(ii) The linear equation

$$
H(x, y, \lambda) \mu(x, y, \lambda)=\left\{\frac{\partial F}{\partial \lambda}, F\right\}(x, y, \lambda)
$$

has a solution $\mu(x, y, \lambda)$ for every $(x, y, \lambda) \in \mathcal{O}$.

Then $D^{\prime}$ is an integrable Lagrangian differential system. 
Proof. Assume that $I \ni t \mapsto(x(t), y(t), \lambda(t))$ is a smooth solution of (1). By differentiation of

$$
\frac{\partial F}{\partial \lambda^{k}}(x(t), y(t), \lambda(t))=0, \quad 1 \leq k \leq m,
$$

with respect to $t$, we obtain an implicit equation for $\mu \in \mathbb{R}^{m}$ (which has a sense of $\dot{\lambda}$ ),

$$
\begin{aligned}
0= & \sum_{i} \frac{\partial^{2} F}{\partial \lambda_{k} \partial \lambda_{i}}(x, y, \lambda) \mu_{i}+\sum_{i} \frac{\partial^{2} F}{\partial \lambda_{k} \partial x_{j}} \frac{\partial F}{\partial y_{j}}(x, y, \lambda) \\
& -\sum_{i} \frac{\partial^{2} F}{\partial \lambda_{k} \partial y_{i}} \frac{\partial F}{\partial x_{i}}(x, y, \lambda),
\end{aligned}
$$

which we can rewrite using the Poisson bracket notation:

$$
H \mu=\left\{\frac{\partial F}{\partial \lambda}, F\right\} .
$$

It is easy to see that if we can find a smooth solution of equation (3) with respect to $\mu$, i.e. smoothly depending on $(x, y, \lambda)$ on $\mathcal{O}$, then the system of ordinary differential equations

$$
\begin{aligned}
\dot{x}^{i} & =\frac{\partial F}{\partial y_{i}}(x, y, \lambda), & & 1 \leq i \leq n, \\
\dot{y}_{j} & =-\frac{\partial F}{\partial x^{j}}(x, y, \lambda), & & 1 \leq j \leq n, \\
\dot{\lambda}^{k} & =\mu(x, y, \lambda), & & 1 \leq k \leq m,
\end{aligned}
$$

is locally integrable. In fact using Mather's theorem (cf. [9], p. 185), the assumption of $H$ being a generic matrix on the open domain $\mathcal{O}$ (such matrices form an open and dense set) and the existence of a formal solution of (3) we obtain the result of the theorem.

REMARK 3.1. The condition of Theorem 3.1 is sufficient but not necessary for integrability. Consider the differential system generated by the smooth family $F: \mathbb{R}^{2 n} \times \mathbb{R} \rightarrow \mathbb{R}$,

$$
F(x, y, \lambda)=\Phi^{k}(x, y, \lambda)+\phi(x, y) \Phi^{2}(x, y, \lambda), \quad \frac{\partial \Phi}{\partial \lambda}(0,0,0) \neq 0 .
$$

One may check, by Theorem 3.1, that the corresponding differential system is integrable if $d \phi(0,0) \neq 0$. In fact this system is equivalently generated by

$$
\widetilde{F}(x, y, \lambda)=\lambda^{k}+\phi(x, y) \lambda^{2},
$$


so we find equation (3) in the form

$$
\left(k(k-1) \lambda^{k-2}+2 \phi(x, y)\right) \mu=\left\{\frac{\partial F}{\partial \lambda}, F\right\}=0,
$$

and the matrix

$$
H:(x, y, \lambda) \mapsto k(k-1) \lambda^{k-2}+2 \phi(x, y)
$$

is transversal to $\Sigma_{1}$ if $d \phi(0,0) \neq 0$ in some neighbourhood of zero. We easily see that this system is integrable also if the transversality condition is not satisfied for the matrix $H$.

ExAmple 3.1. Consider a particle subject to a simple restoring force whose centre of attraction is allowed to move freely on the circle $x_{1}^{2}+x_{2}^{2}$ $=r^{2}$. The dynamics of the system is described by the implicit Lagrangian differential system generated by the family

$$
F\left(x_{1}, x_{2}, y_{1}, y_{2}, \theta\right)=\frac{1}{2 m}\left(y_{1}^{2}+y_{2}^{2}\right)+\frac{k}{2}\left[\left(x_{1}-r \cos \theta\right)^{2}+\left(x_{2}-r \sin \theta\right)^{2}\right] .
$$

It is easy to check that the system has an unstable differential caustic which is a 2 -dimensional surface $\{(x, y): x=0\}$. We find

and get

$$
\begin{aligned}
\frac{\partial F}{\partial \theta} & =k r\left(x_{1} \sin \theta-x_{2} \cos \theta\right), \\
\frac{\partial^{2} F}{\partial \theta^{2}} & =k r\left(x_{1} \cos \theta+x_{2} \sin \theta\right),
\end{aligned}
$$

$$
H:(x, y, \theta) \mapsto k r\left(x_{1} \cos \theta+x_{2} \sin \theta\right) .
$$

We see that $F$ satisfies both assumptions of Theorem 3.1, so it is an integrable Lagrangian differential system.

REMARK 3.2. The integrability condition (ii) of Theorem 3.1 becomes sufficient for the Lagrangian differential system of Examples 2.1 and 2.2, and its weaker version gives the following condition for the existence of nontrivial singular curves:

$$
\exists \lambda \in \mathbb{R}^{k}, \lambda \neq 0: \quad\left\{\frac{\partial F}{\partial \lambda_{i}}, F\right\}=0, i=1, \ldots k,
$$

which may be rewritten in the form of a system of linear equations

$$
\sum_{j=1}^{k} \lambda_{j}\left\langle y,\left[X_{i}, X_{j}\right](x)\right\rangle=0
$$

or equivalently

$$
\sum_{j=1}^{k} \lambda_{j}\left\{H_{i}, H_{j}\right\}=0
$$


where $H_{i}(x, y)=\left\langle y, X_{i}(x)\right\rangle$. Define $\mathcal{C}=\left\{(x, y) \in T^{*} X: H_{i}(x, y)=0, i=\right.$ $1, \ldots, k\}$. Then $\beta\left(D^{\prime}\right)$ is a conormal bundle of $\mathcal{C}$ in $T^{*} P$, and we have the following result.

Proposition 3.2. The Lagrangian differential system $D^{\prime}$ given by $\left(1^{\prime}\right)$ is an integrable Lagrangian differential system if and only if $\mathcal{C}$ is a coisotropic submanifold of $\left(T^{*} X, \omega_{X}\right)$. In this case all singular curves are integral curves of the coisotropic distribution

$$
\mathcal{C}^{\vdash}=\left\{u \in T\left(T^{*} X\right): \omega_{X}(u, v)=0 \forall v \in T \mathcal{C}\right\} \subset T \mathcal{C} .
$$

Proof. We repeat the arguments from the proof of Theorem 3.1. But in this special case of systems the existence of the integral curve $\gamma$ passing through any point of $D^{\prime}$ is equivalent to the system of equations

$$
\left\{\frac{\partial F}{\partial \lambda_{i}}, F\right\}(x, y, \lambda)=\sum_{j=1}^{k} \lambda_{j}\left\{H_{i}, H_{j}\right\}(x, y)=0,
$$

satisfied for every $(x, y, \lambda) \in \mathcal{O}$. But this is the condition for $\mathcal{C}$ to be a coisotropic submanifold of $(P, \omega)$.

We can easily see that all constant rank vector subbundles of $D^{\prime}$ given by the condition $\{\partial F / \partial \lambda, F\}=0$ are integrable, so they are integrable sub-Lagrangian (isotropic [6]) differential systems.

If $H_{0}(x, y)=\operatorname{det}\left(\left\{H_{i}, H_{j}\right\}\right)(x, y)=0$ defines a hypersurface in $\mathcal{C}$, then the integrable sub-Lagrangian differential systems are defined as constant rank subbundles by the system of equations

$$
\begin{aligned}
\sum_{j=1}^{k} \lambda_{j}\left\{H_{i}, H_{j}\right\} & =0, \quad i=1, \ldots, k, \\
\sum_{j=1}^{k} \lambda_{j}\left\{H_{0}, H_{j}\right\} & =0 .
\end{aligned}
$$

In general, if $\mathcal{C}=\bigcup_{j \in J} \mathcal{C}^{j}$ is a stratification of $\mathcal{C}$ by rank of the matrix $\left(\left\{H_{i}, H_{j}\right\}\right)(x, y)$, then a necessary and sufficient condition for the integrability of a sub-Lagrangian differential system $D^{\prime \prime}$ defined as the subsystem of $D^{\prime}$ described by $\left(1^{\prime}\right)$ is the inclusion

$$
T \mathcal{C}^{j} \cap D^{\prime} \subset D^{\prime \prime}, \quad j \in J .
$$

REMARK 3.3. Theorem 3.1, in an analogous formulation, is also true in a more general situation if $D^{\prime}$ is not Lagrangian. We show easily that it is not true if the genericity hypothesis (i) is dropped. Then smoothness of the solution of (3) may not be obtained. This can be illustrated by the equation $\lambda^{2} \mu=\lambda$, for which there is not even a continuous solution, though the equation has a solution for each $\lambda$. 
REMARK 3.4. Without loss of generality we can reduce our integrability problem to the case of $F$ analytic. By Cramer's rule there is an $m \times m$ matrix $\widetilde{H}$ of real analytic functions such that

$$
\widetilde{H} H=H \widetilde{H}=(\operatorname{det} H) I,
$$

where $I$ denotes the identity matrix. Then we can rewrite equation (3) in the form

$$
(\operatorname{det} H) \mu=\widetilde{H}\left\{\frac{\partial F}{\partial \lambda}, F\right\} .
$$

Hypothesis (ii) of Theorem 3.1 implies that $\widetilde{H}\{\partial F / \partial \lambda, F\}$ vanishes on $\{\operatorname{det} H=0\}$, thus to show that equation (3) has a solution, it is enough to show that each component of the column vector $\widetilde{H}\{\partial F / \partial \lambda, F\}$ is divisible by $\operatorname{det} H$.

Acknowledgements. I would like to thank Bronisław Jakubczyk and Fernand Pelletier for helpful conversations.

\section{References}

[1] V. I. Arnold, S. M. Gusein-Zade and A. N. Varchenko, Singularities of Differentiable Maps, Vol. 1, Birkhäuser, Boston, 1985.

[2] J. W. Bruce, A note on first order differential equations of degree greater than one and wavefront evolution, Bull. London Math. Soc. 16 (1984), 139-144.

[3] I. Ekeland, Discontinuités de champs hamiltoniens et existence de solutions optimales en calcul des variations, Publ. Math. I.H.E.S. 47 (1977), 5-32.

[4] S. Janeczko, Systems of rays in the presence of distribution of hyperplanes, in: Banach Center Publ. 32, Inst. Math., Polish Acad. Sci., 1995, 245-260.

[5] - Hamiltonian geodesics in nonholonomic differential systems, Reports Math. Phys. 40 (1997), 217-224.

[6] - On isotropic submanifolds and evolution of quasicaustics, Pacific J. Math. 158 (1993), 317-334.

[7] J. Kijowski and W. M. Tulczyjew, A Symplectic Framework for Field Theories, Lecture Notes in Physics 107, Springer, 1979.

[8] J. Martinet, Singularities of Smooth Functions and Maps, Cambridge Univ. Press, Cambridge, 1982.

[9] J. N. Mather, Solutions of generic linear equations, in: Dynamical Systems (Salvador, 1971), Academic Press, 1973, 185-193.

[10] R. Montgomery, A survey of singular curves in sub-Riemannian geometry, J. Dynam. Control Systems 1 (1995), 49-90.

[11] J. Śniatycki and W. M. Tulczyjew, Generating forms of Lagrangian submanifolds, Indiana Univ. Math. J. 22 (1972), 267-275.

[12] F. Takens, Implicit differential equations: some open problems, in: Lecture Notes in Math. 535, Springer, 1976, 237-253.

[13] R. Thom, Sur les équations différentielles multiformes et leurs intégrales singulières, Bol. Soc. Brasil. Mat. 3 (1972), 1-11. 
[14] A. Weinstein, Lectures on Symplectic Manifolds, CBMS Regional Conf. Ser. in Math. 29, Amer. Math. Soc., Providence, RI, 1977.

Institute of Mathematics

Warsaw University of Technology

Pl. Politechniki 1

00-661 Warszawa, Poland

E-mail: janeczko@alpha.im.pw.edu.pl

Reçu par la Rédaction le 20.7.1999

Révisé le 3.1.2000 